\title{
RESEARCH
}

Open Access

\section{Locus coeruleus pathology in progressive supranuclear palsy, and its relation to disease severity}

Sanne Simone Kaalund ${ }^{1,2^{*+}}$ (D), Luca Passamonti ${ }^{1,3,4+}$, Kieren S. J. Allinson ${ }^{4}$, Alexander G. Murley ${ }^{1}$, Trevor W. Robbins ${ }^{5}$, Maria Grazia Spillantini ${ }^{1}$ and James B. Rowe $e^{1,2,4,6}$

\begin{abstract}
The locus coeruleus is the major source of noradrenaline to the brain and contributes to a wide range of physiological and cognitive functions including arousal, attention, autonomic control, and adaptive behaviour. Neurodegeneration and pathological aggregation of tau protein in the locus coeruleus are early features of progressive supranuclear palsy (PSP). This pathology is proposed to contribute to the clinical expression of disease, including the PSP Richardson's syndrome. We test the hypothesis that tau pathology and neuronal loss are associated with clinical heterogeneity and severity in PSP. We used immunohistochemistry in post mortem tissues from 31 patients with a clinical diagnosis of PSP (22 with Richardson's syndrome) and 6 control cases. We quantified the presence of hyperphosphorylated tau, the number of pigmented cells indicative of noradrenergic neurons, and the percentage of pigmented neurons with tau-positive inclusions. Ante mortem assessment of clinical severity using the PSP rating scale was available within $1.8( \pm 0.9)$ years for 23 patients.

We found an average 49\% reduction of pigmented neurons in PSP patients relative to controls. The loss of pigmented neurons correlated with disease severity, even after adjusting for disease duration and the interval between clinical assessment and death. The degree of neuronal loss was negatively associated with tau-positive inclusions, with an average of $44 \%$ of pigmented neurons displaying tau-inclusions.

Degeneration and tau pathology in the locus coeruleus are related to clinical heterogeneity of PSP. The noradrenergic deficit in the locus coeruleus is a candidate target for pharmacological treatment. Recent developments in ultra-high field magnetic resonance imaging to quantify in vivo structural integrity of the locus coeruleus may provide biomarkers for noradrenergic experimental medicines studies in PSP.
\end{abstract}

Keywords: Frontotemporal dementia, Locus coeruleus, Progressive supranuclear palsy, Tau, Neurodegeneration, Noradrenalin

\footnotetext{
* Correspondence: ssk42@medschl.cam.ac.uk

†Sanne Simone Kaalund and Luca Passamonti contributed equally to this work.

${ }^{1}$ Cambridge University Centre for Parkinson-plus and Department of Clinical Neurosciences, University of Cambridge, Robinson Way, Cambridge CB2 0SZ, UK

${ }^{2}$ Danish Research Centre for Magnetic Resonance (DRCMR), Centre for Functional and Diagnostic Imaging Research, Copenhagen University Hospital Hvidovre, Hvidovre, Denmark

Full list of author information is available at the end of the article
}

(c) The Author(s). 2020 Open Access This article is distributed under the terms of the Creative Commons Attribution 4.0 International License (http://creativecommons.org/licenses/by/4.0/), which permits unrestricted use, distribution, and reproduction in any medium, provided you give appropriate credit to the original author(s) and the source, provide a link to the Creative Commons license, and indicate if changes were made. The Creative Commons Public Domain Dedication waiver (http://creativecommons.org/publicdomain/zero/1.0/) applies to the data made available in this article, unless otherwise stated. 


\section{Introduction}

The locus coeruleus is the principal source of noradrenaline, with diverse influences on arousal, behaviour, movement, and cognition [4]. The locus coeruleus is vulnerable to neurodegeneration in several diseases including Alzheimer's disease, Parkinson's disease, and progressive supranuclear palsy (PSP) $[1,11,24,28,50,56]$. Here, we focus on the neuropathology of the locus coeruleus in PSP, a complex parkinsonian syndrome characterised by postural instability, falls, oculomotor impairment, cognitive, and behavioural changes. We test the hypotheses that, in patients with clinical and pathologically confirmed diagnosis of PSP, there is severe degeneration and tau-pathology in the locus coeruleus, and that the degree of noradrenergic cell loss relates to clinical severity.

The locus coeruleus is located in the posterior margin of the rostral pons, near the lateral floor of the fourth ventricle. Despite its small size, with only tens of thousands of neurons in adult humans [1, 28, 34, 37, 40, 48], the locus coeruleus sends widespread projections to the neocortex, thalamus, and sub-cortical areas, sparing the majority of the striatum. Its connectivity enables a concerted release of noradrenaline in multiple target areas with modulatory effects on several physiological and cognitive functions including arousal, vigilance, sleep, attention, workingmemory, and adaptive behaviour (reviewed by [4, 14]). Many of these functions are affected by PSP, over and above the PSP's classical movement disorder. The motor and cognitive symptoms of PSP are not relieved by standard anti-parkinsonian medications including dopaminergic therapies. However, there is evidence, from studies in Parkinson's disease, that cognitive and behavioural deficits associated with locus coeruleus' degeneration can be partially restored by noradrenergic therapies $[8,27]$, raising the possibility of noradrenergic treatments in PSP.

At the neuropathological level, the degeneration of the locus coeruleus in PSP includes neuronal loss and the presence of neuronal and glial inclusions of hyperphosphorylated 4-repeat isoforms of microtubule-associated protein tau $[45,55]$. In humans, the noradrenergic neurons of the locus coeruleus contain a pigment called neuromelanin. Neuromelanin is synthesised through complex pathways involving iron- and copper-mediated oxidation of cytosolic catecholamines into quinones. These accumulate with proteins and lipids into autophagic organelles, which fuse with lysosomes to form neuromelanin organelles [51, 60]. Although less is known about the synthesis of neuromelanin in the locus coeruleus than substantia nigra, the pathways are likely to be similar [51], and may be neuroprotective [31, 47]. The de-pigmentation of the locus coeruleus in PSP resulting from loss of neuromelanincontaining neurons is so extensive that it is visible on gross post mortem examination of the brain. Recently, the development of high-resolution magnetic resonance imaging
(MRI) sequences [44], sensitive to the paramagnetic features of neuromelanin [58], has renewed the interest in developing biomarkers for assessing the in vivo degeneration of the locus coeruleus in neurodegenerative diseases including PSP [7]. However, before these MRI methods can be further developed, it is necessary to quantify the neuronal loss in the LC ex vivo and determine whether this pathology relates to other neuropathological aspects in PSP such as the proportion of tau-positive inclusions, and to clinical severity.

Therefore, we quantified the locus coeruleus neuropathology in complementary ways. First, we estimated the total number of pigmented neurons post mortem in PSP patients in relation to a group of controls of similar age. Second, we estimated the number of pigmented neurons in the locus coeruleus that manifested neuronal inclusions comprising aggregated hyperphosphorylated tau. Third, we tested the correlations between pathological and clinical ratings. We confirm the severe loss of locus coeruleus neuron number, and a high rate of tau inclusions [20,39], with a correlation between ante mortem disease severity (adjusting for time between latest clinical assessment and death), and the severity of neuronal loss in the locus coeruleus.

\section{Materials and methods}

Brainstem tissue from patients and controls was obtained through the Cambridge Brain Bank at the Cambridge University Hospitals NHS Trust, UK (under the ethically approved protocol for "Neurodegeneration Research in Dementia") and normative cognitive data from the PiPPIN cohort ("Pick's disease and progressive supranuclear palsy prevalence and incidence study" [17]). Thirty-one patient donations were received between 2010 and 2017 from patients with a clinical and pathological diagnosis of PSP. The available fixed tissue blocks for two PSP-cases did not include the entire locus coeruleus so for these two we only report their percentage of pigmented neurons positive for tau-inclusions. Moreover, in two other cases, no immunohistochemistry could be performed because tissue sections detached from the glass slide.

The neuropathological diagnosis of PSP was based on the National Institute of Neurological Disorders and Stroke (NINDS) criteria. Clinical diagnoses were made according to the revised MDS 2017 criteria (Höglinger et al., 2017), based on the final clinical review (see Gazzina et al. for details [22]). This led to diagnoses of probable PSP-Richardson's syndrome in $n=22$, possible PSP with predominant speech/language disorder (PSP-SL) in $\mathrm{n}=2$, and possible PSP with predominant corticobasal syndrome (CBS) in $n=7$.

Many of the PSP patients had also participated in longitudinal cohorts studies at the Cambridge Centre for Frontotemporal Dementia and Related Disorders [17, 
30]. Clinical, cognitive, and behavioural assessments included the PSP rating scale (PSPRS) [23], revised Addenbrooke's cognitive examination (ACE-R) [36], Mini Mental State Examination (MMSE) [21], and revised Cambridge Behavioural Inventory (CBI-R) [52]. The PSPRS was available within 3 years of death for $n=19$ cases of PSP-Richardson's syndrome, $n=2$ PSP-CBS and $\mathrm{n}=1$ PSP-SL. For control tissue, we examined six Brain Bank donors with no history of neurological or psychiatric illness and of similar age and sex as PSP cases. Ante mortem clinical and cognitive data for control brain donors were not available. However, we compared the cognitive profile of the PSP patients to a control population of elderly individuals $(n=60)$ recruited in a regional epidemiological study [17].

Group ages, brain weight, and cognitive scores were compared with two-samples independent $\mathrm{t}$-test (Tables 1 and 2). Sex differences between groups were tested using Fisher's exact test (Table 1).

\section{Tissue processing and immunohistochemistry}

The brain was removed at autopsy and the left cerebral hemisphere, left hemi-brainstem, and left cerebellum were fixed in 10\% neutral buffered formalin for 2-3 weeks. For diagnostic purposes immunohistochemistry was performed on sections from brainstem, subcortical, cerebellum, archicortex and cerebral regions to identify the characteristic protein aggregates and pathological features of PSP and confirm the diagnosis post mortem. The initial diagnostic immunohistochemistry had used antibodies targeting Tau11/57 (from 2010 to mid-2016), or AT8 (from mid-2016 to 2017; MN1020, Thermo Scientific, USA). To exclude significant co-pathologies, additional immunohistochemistry included hippocampal and temporal neocortical beta-amyloid (Clone 6F/3D, M0872, Dako, Denmark). For those cases who had come into the brain bank between 2010- mid-2016 $(n=24)$ the presence of lewy bodies was assessed in all areas on the H\&E slides, and screening for ubiquitin (Z0458, Dako, Denmark) in hippocampal and temporal neocortex. This ubiquitin antibody also stained TDP-43 inclusions. Cases dying after mid-2016 $(n=7)$ were stained for medullary alpha-synuclein (SA3400, Enzo life sciences, USA) and hippocampal and temporal neocortical TDP-43 (TIP-PTD-P02, Cosmo Bio Co LTD, Japan).

To ensure that the entire locus coeruleus was available for the estimation of numbers of neurons, the diagnostic blocks containing the pons and lower midbrain were retrieved and added to the fixed samples containing the locus coeruleus. Brain tissue were embedded in paraffin and cut at $10 \mu \mathrm{m}$ on a rotary microtome and mounted on SuperFrost Plus ${ }^{\text {Th }}$ Adhesion Microscope Slides (J1800AMNZ, ThermoFischer Scientific, USA). For each individual case, every 100th section was immunostained for hyperphosphorylated tau (AT8) to visualise pathological tau aggregates. In brief, sections were deparaffinised in xylene and hydrated in progressively decreasing concentrations of alcohol and rinsed in running water. Antigen retrieval was performed at room temperature by incubating for $5 \mathrm{~min}$ in formic acid (33015-2.5 L-M, Sigma-Aldrich, Germany), and blocked in 5\% normal rabbit serum, before incubating with the primary antibody (AT8 1:500, MN1020, Thermo Scientific, USA) for $1 \mathrm{~h}$ at room temperature. Sections were subsequently incubated with Polyclonal Rabbit Anti-Mouse Immunoglobulins/Biotinylated (E0354, Dako, Denmark) in 10\% human serum and developed using the VECTASTAIN ELITE ABC Kit Avidin Biotinylated HRP Complex (PK-

Table 1 Demographic and clinical characteristic of patients with PSP, and pathology-controls

\begin{tabular}{|c|c|c|c|c|c|}
\hline & $\operatorname{PSP}(n=31)$ & PSP-RS $(n=22)$ & Variant PSP $(n=9)$ & Controls $(n=6)$ & p-value Control - PSP \\
\hline \multicolumn{6}{|l|}{ Age (years) at Death } \\
\hline Mean \pm SD & $75.4 \pm 7.4$ & $73.2 \pm 7.1$ & $80.7 \pm 5.1$ & $70 \pm 5.6$ & n.s. ${ }^{a}$ \\
\hline (Range) & $(63-90)$ & $(63-90)$ & $(73-90)$ & $(62-77)$ & \\
\hline Number (\%) of males & $19(61 \%)$ & $14(63 \%)$ & $5(56 \%)$ & $2(33 \%)$ & n.s.b \\
\hline \multicolumn{6}{|c|}{ Age (years) at symptom onset } \\
\hline Mean \pm SD & $71.5 \pm 8.3$ & $66.0 \pm 8.2$ & $73.7 \pm 5.5$ & - & - \\
\hline (Range) & $(51-86)$ & $(51-86)$ & $(68-86)$ & & \\
\hline \multicolumn{6}{|l|}{ Disease duration (years) } \\
\hline Mean \pm SD & $6.9 \pm 2.9$ & $7.0 \pm 2.8$ & $6.9 \pm 3.3$ & - & - \\
\hline Range & $(3.1-15.5)$ & $(4.2-15.5)$ & $(3.1-14.3)$ & & \\
\hline \multicolumn{6}{|l|}{ Brain weight (g) } \\
\hline Mean \pm SD & $1196 \pm 142.7$ & $1193 \pm 151.5$ & $1209 \pm 117.7$ & $1222 \pm 47.8$ & n.s. ${ }^{a}$ \\
\hline Range & (960-1500) & (960-1500) & (1079-1400) & $(1153-1263)$ & \\
\hline
\end{tabular}

PSP progressive supranuclear palsy, SD standard deviation, n.s. not significant, $p>0.05$ uncorrected, by ${ }^{\text {a }}$ Student's t-test or ${ }^{\mathrm{b}}$ Fischer's exact test, Subscript to $\mathrm{t}$ is degrees of freedom 
Table 2 Demographic and cognitive characteristics of the PSP patients and healthy controls from the "PiPPIN" regional epidemiological study

\begin{tabular}{|c|c|c|c|c|c|}
\hline & PSP patients $(n=31)$ & PSP-RS $(n=22)$ & Variant PSP $(n=9)$ & Controls (PiPPIN) $(n=60)$ & $p$-value Control - PSP \\
\hline \multicolumn{6}{|l|}{ Age at testing } \\
\hline Mean \pm SD & $73.3 \pm 7.7$ & $71.02 \pm 7.3$ & $79.9 \pm 5.6$ & $71.4 \pm 6.0$ & n.s. ${ }^{a}$ \\
\hline Number (\%) of males & 19 (61\%) & $14(63 \%)$ & $5(56 \%)$ & $30(50 \%)$ & n.s. ${ }^{b}$ \\
\hline \multicolumn{6}{|l|}{ Interval to death (year) } \\
\hline Mean \pm SD & $1.8 \pm 0.9$ & $2.2 \pm 0.8$ & $1.8 \pm 0.8$ & - & - \\
\hline PSPRS & $n=23$ & $n=19$ & $n=3$ & & \\
\hline Mean \pm SD & $48.9 \pm 14.1$ & $47.9 \pm 14.1$ & $55 \pm 15.1$ & $0.32 \pm 0.99$ & $\mathrm{t}_{45}=17.2, p=2.2 \mathrm{e}^{-21}$ \\
\hline \multicolumn{6}{|l|}{ ACER } \\
\hline Mean \pm SD & $64.2 \pm 16.0$ & $65.0 \pm 15.0$ & $62.4 \pm 19.2$ & $95.7 \pm 4.4$ & $\mathrm{t}_{89}=14.3, p=9.5 \mathrm{e}^{-25}$ \\
\hline CBI-R & $n=24$ & $n=17$ & $n=7$ & & \\
\hline Mean \pm SD & $84.5 \pm 52.7$ & $83.6 \pm 52.1$ & $86.4 \pm 52.7$ & $6.8 \pm 7.3$ & $\mathrm{t}_{82}=11.3, p=2.6 \mathrm{e}^{-18}$ \\
\hline \multicolumn{6}{|l|}{ MMSE } \\
\hline Mean \pm SD & $21.9 \pm 4.8$ & $22.3 \pm 4.4$ & $21 \pm 5.8$ & $29.4 \pm 0.9$ & $\mathrm{t}_{89}=-11.6, p=1.4 \mathrm{e}^{-19}$ \\
\hline
\end{tabular}

For PSPRS and CBI-R the $\mathrm{n}$ above the mean indicates the number of patients from which data was available. The controls were healthy, age and sex-matched controls from a regional population cohort, PiPPIN [17]. PSP progressive supranuclear palsy, PSP-RS PSP Richardson's syndrome, PiPPIN “Pick's disease and progressive supranuclear palsy prevalence and incidence study" [17], PSPRS PSP rating scale, ACER revised Addenbrooke's cognitive examination, CBI-R revised Cambridge Behavioural Inventory, MMSE Mini Mental State Examination, $S D$ standard deviation, n.s. not significant, $p>0.05$, by ${ }^{\mathrm{a}}$ Student's t-test or ${ }^{\mathrm{b}}$ Fischer's exact test. Subscript to $t$ is degrees of freedom. Uncorrected $p$-values

6100, VECTOR laboratories, USA) to yield a purple reaction product. Sections were counter stained with Vector Methyl green (H-3402-500, Vector laboratories, USA) to allow good visual discrimination between cell nuclei, tau aggregates, and endogenous neuromelanin (Fig. 1).

\section{Quantification of pigmented neurons in locus coeruleus}

A 2D design was used to estimate the total number of pigmented neurons (StereoInvestigator 11.0 64bit, Microbrightfield, USA) using an Olympus BX-53 microscope coupled with a Prior H128 computer-controlled $\mathrm{x}-\mathrm{z}-\mathrm{y}$ motorized stage and a high sensitivity Hitachi 3CCD video camera system. The locus coeruleus was outlined at $4 \mathrm{x}$ magnification and counting within 100\% of that area was performed under a $20 \mathrm{X}$ objective. Pigmented neurons were defined by the presence of neuromelanin, which appears as brown granular staining within the cytoplasm of medium and large neurons in the locus coeruleus. When an AT8-positive aggregate was present in these pigmented neurons, the neuron was counted as tau immunoreactive. A neuron was counted when either the nucleolus or a well-defined nucleus was in focus (Fig. 1).

The number of pigmented neurons was calculated by multiplying the number of neurons counted in the serial sections by 100 (the sampling frequency). As sections were $10 \mu \mathrm{m}$ and we counted pigmented neurons in the entire cross-sectional area of locus coeruleus on each section, the height and area fractions were 1 . Using this sampling scheme, an average of 9 sections were counted in controls, and an average of 7 sections in PSP. The proportion of neurons with hyperphosphorylated tauinclusions was determined by dividing the number of pigmented neurons positive for hyperphosphorylated tau-inclusions by the total number of pigmented neurons.

\section{Quantification of total tau burden in the locus coeruleus}

The overall burden of pathological tau in the locus coeruleus was estimated as the percentage of pixels associated with tau staining within the locus coeruleus area, using the build-in "positive pixel count" in QuPath (0.2.0.m5). Micrographs of the locus coeruleus were acquired from the serial sections used for neuronal estimates under a $20 \mathrm{X}$ objective. A series of images were uploaded in QuPath, next, the area of the locus coeruleus was manually outlined, and stain vectors for neuromelanin, tau, and background signal were adjusted for each section. The percentage of 'tau' positive pixels within the region of interest was measured using the following settings in the "positive pixel count"-algorithm: Down-sample factor $=1.0$, Gaussian sigma $=2$, neuromelanin threshold (negative) $=0.2$, tau threshold (positive) $=$ 0.2 .

We used weighted least-square regression to examine the correlation between the number of pigmented neurons and PSPRS, ACER, MMSE, or CBI scores, and the relationship between the percentage of pigmented neurons with tau-inclusion and PSPRS score. To adjust for the difference in time between the last clinical assessment and death in relation to total disease duration, we 

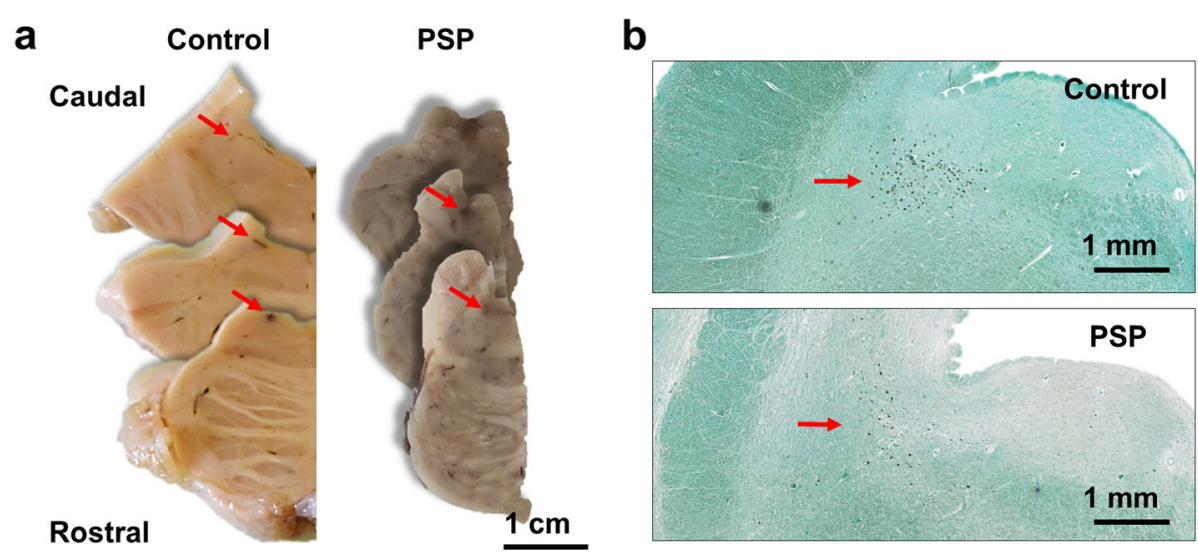

$\mathbf{C}$
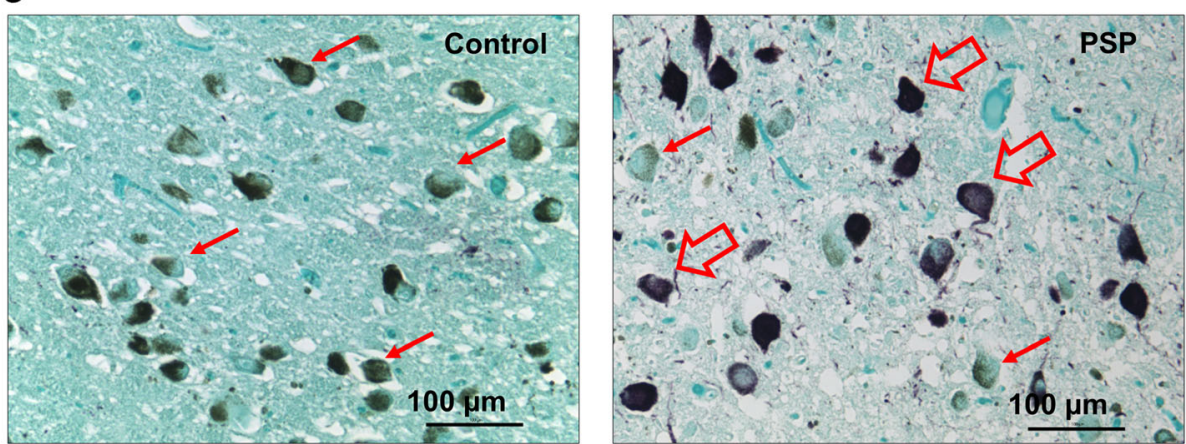

Fig. 1 Representative images of locus coeruleus in control and PSP cases. a Photographs of pons sections including the locus coeruleus (arrows). b Micrographs showing the location of locus coeruleus near the floor of the 4th ventricle in a control and PSP case (red arrows). Stitched images from micrographs taken using a 4X objective. c Micrographs taken using a 20X objective, red arrows indicate representative examples of pigmented neurons of the locus coeruleus and hollow red arrows indicate examples of pigmented neurons with tau-inclusions. $\mathbf{b}$ and $\mathbf{c}$ Sections were stained for hyperphosphorylated tau (AT8) in purple using methyl green as counterstain, endogenous neuromelanin shown in brown

included the time between clinical assessment and death as percent of disease duration (100*(1-"interval between testing and death"/"disease duration")) (these values represented weights in the linear regression analyses). To test for an association between the number of pigmented neurons and the macroscopic scoring of locus coeruleus pallor, we used analysis of variance (ANOVA). Finally, Pearson's correlations were used to test for associations between (i) the total number of pigmented neurons and the percentage of pigmented neurons with tau-inclusions, (ii) the pathological measures, disease duration, disease severity or other cognitive and neuropsychiatric measures, and (iii) total tau burden. A $p$-value $<0.05$ was considered significant.

\section{Results}

Table 1 summarises the clinical, demographic, and gross pathological characteristics of the brain donors. There were no significant differences in age, sex or brain weight between the PSP and pathology control cases. The control group from the PiPPIN epidemiological study was similar to the PSP group in terms of age and sex. However, as expected, PSP patients scored higher on the PSPRS and CBI, and lower on the ACE-R and MMSE than controls from the PiPPIN study (Table 2).

Five of the 31 patients in the present study, 16\%, had co-pathology; 4 with amyloid beta, 0 with alphasynuclein, 0 with TDP-43 and 1 with ubiquitin-poisitve TDP-43- and tau-negative inclusions in the hippocampus. The level of co-pathology was within limits of what can be expected as age related pathology in all cases.

The mean total number of pigmented neurons in the locus coeruleus was $50.6 \times 10^{3}$ in controls (SD $1.4 \times 10^{3}$ ) and $26.2 \times 10^{3}$ in PSP patients (SD $1.4 \times 10^{3}$ ). This $49 \%$ reduction of pigmented neurons in PSP patients was significant $(\mathrm{t}=3.96, \mathrm{df}=33, p=0.0004$, Fig. $2 \mathrm{a}$ ).

Disease severity, as measured by total-PSPRS, was negatively correlated to the number of pigmented neurons $(\mathrm{F}(1,19)=5.9, \mathrm{t}=-2.4, p=0.026$; Fig. $2 \mathrm{~b})$. The total number of pigmented neurons in the locus coeruleus did not correlate with disease duration (Pearson's correlation, $\mathrm{r}=-0.22, p=0.26$ ) or age (Pearson's correlation, $\mathrm{r}=-0.24, p=0.16)$. We did not observe a significant correlation between the number of pigmented neurons and $\operatorname{ACER}(\mathrm{F}(1,28)=0.72, p=0.40), \operatorname{MMSE}(\mathrm{F}(1,28)=$ $0.99, p=0.33)$ or $\mathrm{CBI}(\mathrm{F}(1,21)=1.45, p=0.24)$. The 

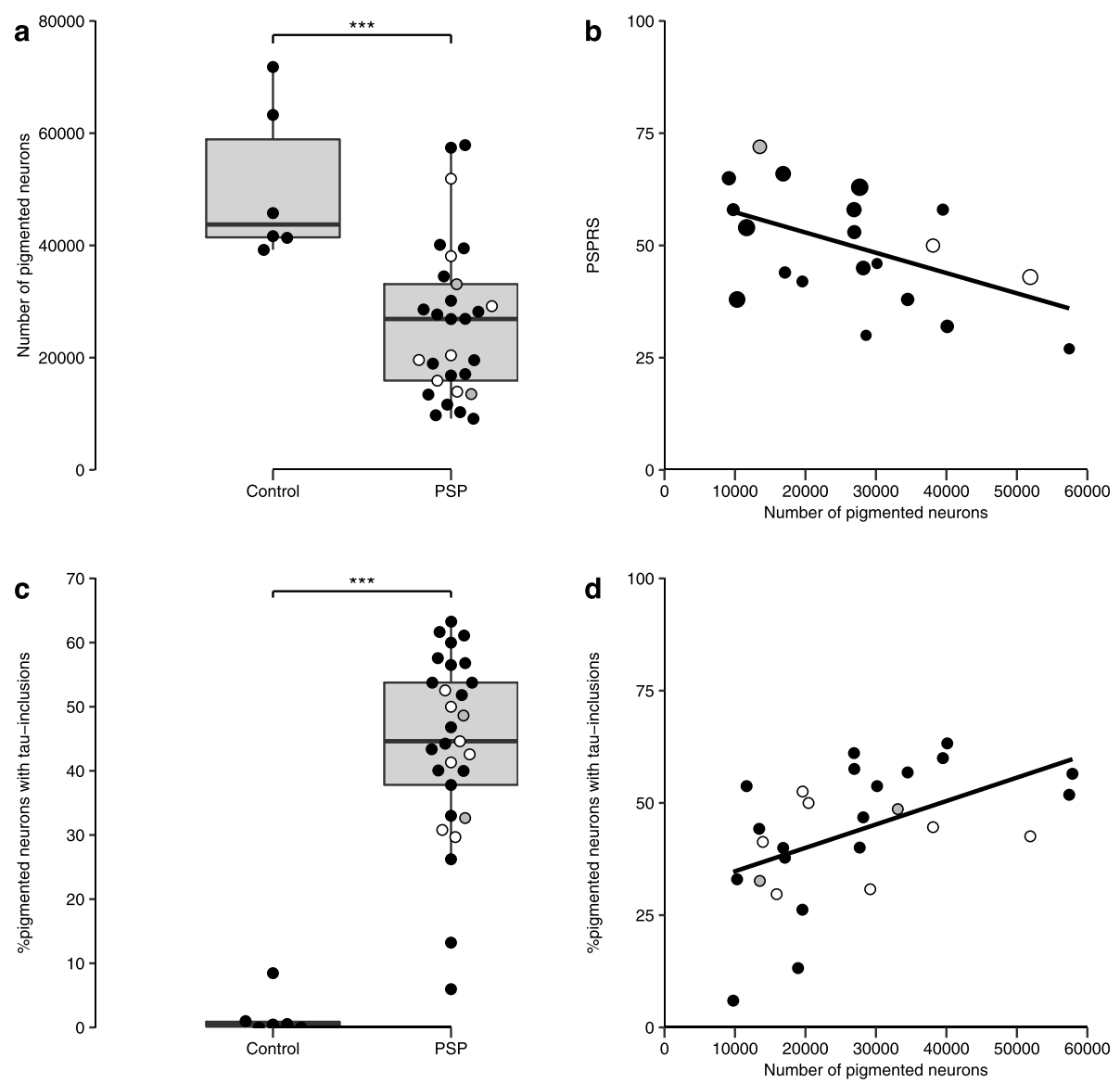

Fig. 2 Loss of pigmented neurons in the locus coeruleus in PSP. a Combined scatter and boxplots showing the total number of pigmented neurons in the left locus coeruleus in controls and patients. $\mathbf{b}$ There was a negative correlation between the number of pigmented neurons and the PSP rating scale (PSPRS) ( $n=20$, weighted least-square regression $F(1,19)=5.9, p=0.026)$ ). The dot size for each individual scales with interval from assessment to death as a function of disease duration. $\mathbf{c}$ The percentage of pigmented neurons with tau-inclusions in controls and PSP. $\mathbf{d}$ The positive correlation between the number of pigmented neurons and number of pigmented neurons with tau-inclusions. Each dot represents an individual case, and for PSP cases (a-d), different colours of the dot represent specific PSP clinical phenotypes, i.e., black - probable PSP Richardson's Syndrome, grey - possible PSP-SL, white - possible PSP-CBS. The lower and upper hinges of the grey boxes show the 25th and 75th percentiles, while the horizontal bar represent the median values. Whisker-plots display the range of data within 1.5 of the inter-quartile range, indicating that no extreme outlying values were observed. The linear regression lines are plotted in panel $\mathbf{b}$ and $\mathbf{d}$

macroscopic scoring of locus coeruleus pallor was not significantly related to the microscopic counting of the number of pigmented cells $(\mathrm{F}(2,21)=1.37, p=0.28)$.

The pigmented neurons positive for hyperphosphorylated tau were observed in four out of six controls, but the percentage of such neurons relative to total number of pigmented neurons was only $3 \%$ (range 0.5 to $9 \%$ ). In PSP, the average percentage of pigmented neurons positive for hyperphosphorylated tau was 44\% (range 6 to $63 \%$ ). The increase in the number of pigmented neurons with tau-inclusions in PSP was significant $(t=-7.26$, $\mathrm{df}=33, p=2.5 \mathrm{e}^{-8}$ ) (Fig. 2c). The percentage of pigmented neurons with tau-inclusions was not associated with PSPRS $(F(1,19)=0.15, \mathrm{t}=0.39, p=0.71)$. However, the percentage of neurons positive for hyperphosphorylated tau positively correlated with the total number of neurons in PSP patients (Pearson's $\mathrm{r}=0.51, p=0.007$ ) (Fig. 2d). Last, the number of pigmented neurons was not significantly correlated with total tau burden in the locus coeruleus (Pearson's $r=0.096, p=0.66$ ).

\section{Discussion}

This study demonstrates severe neurodegeneration in the locus coeruleus in PSP, with an average $49 \%$ reduction in the total number of pigmented neurons that are the principal source of noradrenaline to the brain. The total number of noradrenergic and neuromelanincontaining cells in PSP correlated negatively with disease severity, with fewer cells in those patients who had more severe disease at the last ante mortem clinical assessment. However, neuronal loss was not associated with age or disease duration, which suggests a possible role of 
the locus coeruleus degeneration in mediating clinical severity rather than simply reflecting an age-related effect or a consequence of a more prolonged disease course. The average percentage of pigmented neurons with hyperphosphorylated tau-inclusions in PSP was 44\%, but this fraction was lower in PSP patients with more severe total neuronal loss. This suggests either a non-linear dynamic relationship between tau-aggregation and cell death, or the presence of a subset of pigmented neurons with low susceptibility to tau aggregation and death.

The average $49 \%$ loss of pigmented neurons that we found in this study is highly consistent with the $53 \%$ and $51 \%$ reduction of medium-to-large neurons in the locus coeruleus reported in two previous studies [33, 39]. However, another semi-quantitative study, in which neurons positive for tyrosine hydroxylase were counted in single sections in the upper pons, did not report a significant loss of neurons in the locus coeruleus in PSP [20]. The differences across the studies may be due to the focus on the rostral pons rather than on the entire locus coeruleus, and to other possible differences in how the noradrenergic cells were defined and counted. In the present study, the neuromelanin-containing neurons were assumed to represent the entire noradrenergic neuronal population in the locus coeruleus. This is because the presence of the neuromelanin in the locus coeruleus cells is a consequence of excess catecholamines in the cytosol, which is in turn linked to the synthesis of the noradrenaline and its packing in synaptic vesicles $[3,4]$.

Another way to identify the locus coeruleus cells producing noradrenaline is via the immuno-staining for tyrosine hydroxylase, the enzyme that converts L-tyrosine to LDOPA before the conversion of L-DOPA to noradrenaline. Immunohistochemistry against the dopamine betahydroxylase, the enzyme that transform dopamine into noradrenaline, can also be used. These two biochemical markers are expressed by the same neuronal population [15] i.e., the noradrenergic cells that constitutes more than $95 \%$ of neurons in the locus coeruleus [2,39]. However, a proportion of tyrosine hydroxylase positive neurons, which are more numerous in the rostral than caudal pons, lack pigmentation $[2,15,25]$. The number of such neurons is limited in relation to the total neuronal number in the locus coeruleus $[2,15,25]$. Therefore, despite potential differences in the methods for identifying noradrenergic neurons, the findings from different pathological cohorts were in keeping with a significant reduction of noradrenergic neurons in PSP.

Our second hypothesis was that the loss of LC pigmented neurons relates to disease severity, measured via the PSPRS. This was confirmed. The PSPRS is the most widely used clinical scale of disease severity and progression in PSP, in observational studies and clinical trials. It encompasses diverse domains of symptoms and signs that are typically endorsed by PSP patients and their carers, including behavioural, gait, mobility, bulbar, limb, and oculomotor problems. Other disorders and even normal aging can influence the total PSPRS score [23], but in the context of PSP clinical syndromes, the PSPRS has high internal consistency and replicability across different countries (e.g., there is a typical 10-12 points decline per year in Richardson's syndrome) [9, 23]. The total score and domains of the PSPRS have been associated with PSP-related regional atrophy in the brainstem $[18,26,49$, 54] but not yet related to specific brainstem nuclei as the locus coeruleus [38] or in vivo tau pathology as measured via positron-emission tomography $[19,54]$.

The association between the locus coeruleus integrity and disease severity raises the possibility of noradrenergic treatment strategies [41]. One study reported that alpha 2 noradrenergic receptor antagonism did not alleviate motor symptoms in PSP [43]. However, there is more favourable evidence, from studies in Parkinson's disease (PD), that the noradrenalin reuptake inhibitor atomoxetine can improve motor control and executive functions in a subset of patients $[8,27,57]$. The relationship between the loss of pigmented neurons in the locus coeruleus and disease severity calls for a stratified approach in clinical trials, which might exploit emerging imaging biomarkers of LC structural integrity. Currently, non-invasive methods to quantify the in vivo LC structural integrity, via the susceptibility of the MRI signal to the paramagnetic effects of neuromelanin, are emerging as promising tools for neurodegenerative disorders, especially at ultra-high field $[5,44]$. The association between post mortem depigmentation and the neuromelanin-related MRI signal in the LC may therefore provide support for in vivo biomarkers for noradrenergic dysfunction in PSP. This could facilitate the monitoring of the disease progression and treatment response [7, 42].

Not all pigmented neurons in the locus coeruleus are equally affected by PSP. On average, $44 \%$ of the pigmented neurons had tau-inclusions. This accords with a semi-quantitative study showing tau inclusions in $42 \%$ of the noradrenergic neurons [20], and the presence of tau inclusions in $77 \%$ of the noradrenergic neurons counted using stereology [39]. The current cohort was larger than those employed in previous studies and found high variability, $6-63 \%$, of LC neurons having tau-inclusions. This implies that differences between our findings and previous results might depend on the use of different sample sizes with variable estimates. Differences in the analytical procedures used or in the demographic characteristics of the cohorts may also have contributed to the different percentages of tau-positive neurons across studies. We found a significant positive correlation between the percentage of pigmented neurons with tauinclusions and the total number of pigmented neurons. 
However, the percentage of the pigmented neurons with tau-inclusions was not associated with disease severity. These results are consistent with the hypothesis that the accumulation of tau precedes and contributes to the neuronal death [53], with differential susceptibility to cell death with tau-positive neurons as the disease progresses, or as a result of subtypes of pigmented neurons. Dying neurons may also release extra cellular neuromelanin and trigger microglia activation and inflammation, as observed in the substantia nigra in Parkinson's disease $[29,35]$ and consistent with the co-localisation of activated microglia and tau pathology in PSP [32]. Nigral microglia are able to phagocytose and degrade neuromelanin, but in doing so release hydrogen peroxide and pro-inflammatory cytokines [59], which may lead to further neuronal death. Neurodegeneration within the locus coeruleus could thus be augmented through similar tauindependent mechanisms.

The current data can also be compared to those reported in the tauopathy of Alzheimer's disease, in which the neurofibrillary tangles in the locus coeruleus are present at the pre-symptomatic Braak stages I and II [10, 12, 13, 28, 48]. In Alzheimer's there is severe neuronal loss from Braak stage III onwards [1, 13, 28, 48]: from 13 to $30 \%$ in mild cognitive impairment and Braak stage I-III [1, 28], to $45-50 \%$ in Braak stages IV-V [1, 28, 40], and 70-75\% at Braak stages V and VI [1, 39]. As in PSP, cell loss in the locus coeruleus correlates with ante mortem global cognition in Alzheimer's disease $[1,28]$. We find an average loss of $49 \%$ of pigmented neurons in PSP, but the casespecific loss ranged widely, in proportion to the severity of disease (PSP rating scale). Noradrenergic projections from the locus coeruleus reach widespread cortical and subcortical targets, that are differentially affected by Alzheimer's disease, PSP, and other degenerative disorders. We suggest that whereas the degree of noradrenergic loss in Alzheimer's and PSP is similar, the functional consequences are in part determined by additional pathology in the regions receiving noradrenergic inputs. In contrast to the correlation between the percentage of pigmented neurons with tau-inclusions and neuronal loss we observed in PSP, the percentage of noradrenergic neurons with intracellular neurofibrillary tangles does not correlate with the total number of locus coeruleus neurons in Alzheimer's disease [13]. This difference may depend on the different tau isoforms present in AD and PSP. Future studies addressing the causal relationships between tau inclusions, neuronal loss, noradrenergic signalling in the locus coeruleus in PSP and other neurodegenerative disorders will help to refine future interventional studies.

The present study has several limitations. First, as the selection of cases was based on the post mortem neuropathological diagnosis of PSP, we lack the PSP rating scale in some cases with clinical diagnosis of PSP-CBS.
Second, as the PSP rating scale was necessarily obtained ante mortem, the interval between clinical and cognitive testing and death varied between patients, although we sought to mitigate this issue by including, in our statistical models, the interval between the last assessment and death (in terms of percentage of total disease duration). The association between pigmented neurons and PSP rating scale remained significant with and without this weighting in the statistical model. Third, as we only focused on the locus coeruleus pathology in PSP, we cannot exclude a priori that the association between the PSP rating scale and locus coeruleus pathology reflect a more general association with neurodegeneration at secondary sites, including the targets of LC innervation. Fourth, in the present study we counted the pigmented cells in the locus coeruleus in serial sections selected using systematic sampling. We counted cells on the series of $2 \mathrm{D}$ sections rather than $3 \mathrm{D}$, which is necessary for a true unbiased stereological method. Our estimates are therefore not unbiased and may be skewed towards overestimation as the section thickness is less than the average diameter of pigmented cells [16]. Further, in a 2D fractionator larger particles will have a greater chance of being sampled [46], which may contribute further to an overestimation bias. We estimated the total number of pigmented neurons to 56,000 in the left locus coeruleus in controls. This estimate is larger than what has been reported in a number of stereological studies that estimated the unilateral number of pigmented neurons to range between 16,000-19,000 [1, 28, 34, 37, 40] . However, Theofilas et al. [48] used stereology to estimate the total number of mid- to large neurons in the locus coeruleus in 68 aged individuals from Braak-stage 0 -VI. They observed a mean of 46,500 mid- to large sized neurons in Braak stage 0 , and a total range across all Braak stages from 6674 to 137,910 neurons. Differences in the stereological methodologies e.g. the physical vs. the optical disector or optical fractionator, which cell populations was counted e.g. pigmented [1, 34, 37, 40], tyrosine hydroxylase expressing [28] or size [48], and whether one counts the soma profile or nucleolus may contribute to the discrepancy between studies. Recognizing the inherent potential for biased estimates using a 2D fractionator design, we assume that this bias is constant across subjects and thus does not invalidate the correlations between neuronal numbers and tau-load or disease severity.

\section{Conclusions}

To conclude, we provide evidence for severe degeneration in the locus coeruleus in PSP, and an association between the number of noradrenergic neurons containing neuromelanin and disease severity. However, neuronal loss does not appear to be a simple function of age, 
disease duration or the presence of hyperphosphorylated tau-inclusions. The use of the neuromelanin pigment to identify post mortem the noradrenergic cells in the LC also supports the development of non-invasive in vivo neuroimaging markers that exploit the MRI signal linked to the presence of neuromelanin [6]. The degeneration of the locus coeruleus may be relevant to the development of better stratification procedures in clinical trials. Our study also highlights the importance of considering noradrenergic restoration as a potential treatment for PSP.

\begin{abstract}
Abbreviations
ACE-R: Revised Addenbrooke's cognitive examination; ANOVA: Analysis of variance; CBI-R: Revised Cambridge Behavioural Inventory; CBS: Corticobasal syndrome; MMSE: Mini mental state examination; MRI: Magnetic resonance imaging; NINDS: National Institute of Neurological Disorders and Stroke; PIPPIN: Pick's disease and progressive supranuclear palsy prevalence and incidence study; PSP: Progressive supranuclear palsy; PSPRS: PSP rating scale; PSP-SL: Possible PSP with predominant speech/language disorder
\end{abstract}

\section{Acknowledgements}

We thank the donors and their families.

\section{Authors' contribution}

SSK and LC performed the morphometric experimental work, retrieved ante mortem clinical data, were involved in study design and major contributors in writing the manuscript. KSJA was instrumental in study design, tissue selection and interpretation of the pathological and morphometric data. AGM was instrumental in retrieval of the ante mortem data and provided data from the PIPPIN control cohort. TWR and MGS were instrumental in the study design and contributors in writing the manuscript. JBR was instrumental in the study design, leading the study and a major contributor in writing the manuscript. All authors read and approved the final manuscript.

\section{Funding}

This work was funded by Lundbeck Fonden R232-2016-2333, and R2652017-3722; Wellcome Trust (103838); Medical Research Council (MC_U105597119 \& MC_UU_00005/12 \& SUAG004/051 RG91365), the Holt fellowship, NIHR Cambridge Biomedical Research Centre and Cambridge Brain Bank, and the Cambridge Centre for Parkinson plus. The Cambridge Brain Bank is supported by the NIHR Cambridge Biomedical Research Centre.

\section{Availability of data and materials}

The datasets used are available from the corresponding author on reasonable request.

\section{Ethics approval and consent to participate}

The study ethics was approved by the Health Research Authority, NHS, England (IRAS- 202 802, "Neurodegeneration Research in Dementia"). The PiPPIN (Pick's Disease and Progressive Supranuclear Palsy: Prevalence and Incidence) Study was approved by Cambridge's research ethics committee. The study was conducted in accordance with the 1964 Helsinki declaration.

\section{Consent for publication}

Not applicable.

\section{Competing interests}

The authors declare that they have no competing interests.

\section{Author details}

${ }^{1}$ Cambridge University Centre for Parkinson-plus and Department of Clinical Neurosciences, University of Cambridge, Robinson Way, Cambridge CB2 OSZ, UK. ${ }^{2}$ Danish Research Centre for Magnetic Resonance (DRCMR), Centre for Functional and Diagnostic Imaging Research, Copenhagen University Hospital Hvidovre, Hvidovre, Denmark. ${ }^{3}$ Consiglio Nazionale delle Ricerche (CNR), Istituto di Bioimmagini e Fisiologia Molecolare (IBFM), Milan, Italy.
${ }^{4}$ Cambridge University Hospitals NHS Foundation Trust and the Cambridge Brain Bank, Cambridge, UK. ${ }^{5}$ Department of Psychology and Behavioural and Clinical Neuroscience Institute, University of Cambridge, Cambridge, UK. ${ }^{6}$ Medical Research Council Cognition and Brain Sciences Unit, University of Cambridge, Cambridge, UK.

Received: 16 December 2019 Accepted: 26 January 2020

Published online: 04 February 2020

\section{References}

1. Arendt T, Brückner MK, Morawski M, Jäger C, Gertz H-J (2015) Early neurone loss in Alzheimer's disease: cortical or subcortical? Acta Neuropathol Commun 3:10. https://doi.org/10.1186/s40478-015-0187-1

2. Baker KG, Törk I, Hornung JP, Halasz P (1989) The human locus coeruleus complex: an immunohistochemical and three dimensional reconstruction study. Exp Brain Res 77:257-270. https://doi.org/10.1007/BF00274983

3. Benarroch EE (2018) Locus coeruleus. Cell Tissue Res 373:221-232. https:// doi.org/10.1007/s00441-017-2649-1

4. Berridge CW, Waterhouse BD (2003) The locus coeruleus-noradrenergic system: modulation of behavioral state and state-dependent cognitive processes. Brain Res Rev 42:33-84. https://doi.org/10.1016/501650173(03)00143-7

5. Betts MJ, Cardenas-Blanco A, Kanowski M, Jessen F, Düzel E (2017) In vivo MRI assessment of the human locus coeruleus along its rostrocaudal extent in young and older adults. Neuroimage 163:150-159. https://doi.org/10. 1016/..neuroimage.2017.09.042

6. Betts MJ, Ehrenberg AJ, Hämmerer D, Düzel E (2018) Commentary: locus Coeruleus ablation exacerbates cognitive deficits, neuropathology, and lethality in P301S tau transgenic mice. Front Neurosci 12. https://doi.org/10. 3389/fnins.2018.00401

7. Betts MJ, Kirilina E, Otaduy MCG, Ivanov D, Acosta-Cabronero J, Callaghan MF, Lambert C, Cardenas-Blanco A, Pine K, Passamonti L, Loane C, Keuken MC, Trujillo P, Lüsebrink F, Mattern H, Liu KY, Priovoulos N, Fliessbach K, Dahl MJ, Maaß A, Madelung CF, Meder D, Ehrenberg AJ, Speck O, Weiskopf N, Dolan R, Inglis B, Tosun D, Morawski M, Zucca FA, Siebner HR, Mather M, Uludag K, Heinsen H, Poser BA, Howard R, Zecca L, Rowe JB, Grinberg LT, Jacobs HIL, Düzel E, Hämmerer D (2019) Locus coeruleus imaging as a biomarker for noradrenergic dysfunction in neurodegenerative diseases. Brain 142:2558-2571. https://doi.org/10.1093/brain/awz193

8. Borchert RJ, Rittman T, Passamonti L, Ye Z, Sami S, Jones SP, Nombela C, Vázquez Rodríquez P, Vatansever D, Rae CL, Hughes LE, Robbins TW, Rowe JB (2016) Atomoxetine enhances connectivity of prefrontal networks in Parkinson's disease. Neuropsychopharmacology 41:2171-2177. https://doi. org/10.1038/npp.2016.18

9. Boxer AL, Lang AE, Grossman M, Knopman DS, Miller BL, Schneider LS, Doody RS, Lees A, Golbe LI, Williams DR, Corvol JC, Ludolph A, Burn D, Lorenzl S, Litvan I, Roberson ED, Höglinger GU, Koestler M, Jack CR, Van Deerlin V, Randolph C, Lobach IV, Heuer HW, Gozes I, Parker L, Whitaker S, Hirman J, Stewart AJ, Gold M, Morimoto BH (2014) Davunetide in patients with progressive supranuclear palsy: a randomised, double-blind, placebocontrolled phase 2/3 trial. Lancet Neurol. https://doi.org/10.1016/S14744422(14)70088-2

10. Braak H, Del Tredici K (2011) The pathological process underlying Alzheimer's disease in individuals under thirty. Acta Neuropathol 121:171181. https://doi.org/10.1007/s00401-010-0789-4

11. Braak H, Del Tredici K (2017) Neuropathological staging of brain pathology in sporadic Parkinson's disease: separating the wheat from the chaff. J Park Dis 7:S71-S85. https://doi.org/10.3233/JPD-179001

12. Braak H, Thal DR, Ghebremedhin E, Del Tredici K (2011) Stages of the pathologic process in Alzheimer disease: age categories from 1 to 100 years. J Neuropathol Exp Neurol 70:960-969. https://doi.org/10.1097/NEN. Ob013e318232a379

13. Busch C, Bohl J, Ohm TG (1997) Spatial, temporal and numeric analysis of Alzheimer changes in the nucleus coeruleus. Neurobiol Aging 18:401-406. https://doi.org/10.1016/S0197-4580(97)00035-3

14. Chamberlain SR, Robbins TW (2013) Noradrenergic modulation of cognition: therapeutic implications. J Psychopharmacol 27:694-718. https://doi.org/10. $1177 / 0269881113480988$

15. Chan-Palay V, Asan E (1989) Quantitation of catecholamine neurons in the locus coeruleus in human brains of normal young and older adults and in 
depression. J Comp Neurol 287:357-372. https://doi.org/10.1002/cne. 902870307

16. Coggeshall RE (1992) A consideration of neural counting methods. Trends Neurosci 15:9-13. https://doi.org/10.1016/0166-2236(92)90339-A

17. Coyle-Gilchrist ITS, Dick KM, Patterson K, Rodríquez PV, Wehmann E, Wilcox A, Lansdall CJ, Dawson KE, Wiggins J, Mead S, Brayne C, Rowe JB (2016) Prevalence, characteristics, and survival of frontotemporal lobar degeneration syndromes. Neurology. https://doi.org/10.1212/WNL. 0000000000002638

18. Dutt S, Binney RJ, Heuer HW, Luong P, Attygalle S, Bhatt P, Marx GA, Elofson J, Tartaglia MC, Litvan I, McGinnis SM, Dickerson BC, Kornak J, Waltzman D, Voltarelli L, Schuff N, Rabinovici GD, Kramer JH, Jack CR, Miller BL, Rosen HJ, Boxer AL (2016) Progression of brain atrophy in PSP and CBS over 6 months and 1 year. Neurology 87:2016-2025. https://doi.org/10.1212/WNL. 0000000000003305

19. Endo H, Shimada H, Sahara N, Ono M, Koga S, Kitamura S, Niwa F, Hirano S, Kimura Y, Ichise M, Shinotoh H, Zhang MR, Kuwabara S, Dickson DW, Toda T, Suhara T, Higuchi M (2019) In vivo binding of a tau imaging probe, [ 11 C]PBB3, in patients with progressive supranuclear palsy. Mov Disord 34:744754. https://doi.org/10.1002/mds.27643

20. Eser RA, Ehrenberg AJ, Petersen C, Dunlop S, Mejia MB, Suemoto CK, Walsh CM, Rajana H, Oh J, Theofilas P, Seeley WW, Miller BL, Neylan TC, Heinsen H, Grinberg LT (2018) Selective vulnerability of brainstem nuclei in distinct Tauopathies: a postmortem study. J Neuropathol Exp Neurol 77:149-161. https://doi.org/10.1093/jnen/nlx113

21. Folstein MF, Folstein SE, McHugh PR (1975) "Mini-mental state". A practical method for grading the cognitive state of patients for the clinician. J Psychiatr Res 12:189-198. https://doi.org/10.1016/0022-3956(75)90026-6

22. Gazzina S, Respondek G, Compta Y, Allinson KS, Spillantini MG, MolinaPorcel L, Guasp-Verdaguer M, Moftakhar S, Reich SG, Hall D, Litvan I, Hoeglinger G, Rowe JB (2019) Neuropathological validation of the MDS-PSP criteria with PSP and other frontotemporal lobar degeneration. bioRxiv: 520510. https://doi.org/10.1101/520510

23. Golbe LI, Ohman-Strickland PA (2007) A clinical rating scale for progressive supranuclear palsy. Brain 130:1552-1565. https://doi.org/10.1093/brain/ awm032

24. Grudzien A, Shaw P, Weintraub S, Bigio E, Mash DC, Mesulam MM (2007) Locus coeruleus neurofibrillary degeneration in aging, mild cognitive impairment and early Alzheimer's disease. Neurobiol Aging 28:327-335. https://doi.org/10.1016/J.NEUROBIOLAGING.2006.02.007

25. Iversen LL, Rossor MN, Reynolds GP, Hills R, Roth M, Mountjoy CQ, Foote SL, Morrison JH, Bloom FE (1983) Loss of pigmented dopamine- $\beta$-hydroxylase positive cells from locus coeruleus in senile dementia of alzheimer's type. Neurosci Lett 39:95-100. https://doi.org/10.1016/0304-3940(83)90171-4

26. Josephs KA, Xia R, Mandrekar J, Gunter JL, Senjem ML, Jack CR, Whitwell JL (2013) Modeling trajectories of regional volume loss in progressive supranuclear palsy. Mov Disord 28:1117-1124. https://doi.org/10.1002/mds. 25437

27. Kehagia AA, Housden CR, Regenthal R, Barker RA, Müller U, Rowe J, Sahakian BJ, Robbins TW (2014) Targeting impulsivity in Parkinson's disease using atomoxetine. Brain 137:1986-1997. https://doi.org/10.1093/brain/ awu117

28. Kelly SC, He B, Perez SE, Ginsberg SD, Mufson EJ, Counts SE (2017) Locus coeruleus cellular and molecular pathology during the progression of Alzheimer's disease. Acta Neuropathol Commun 5:8. https://doi.org/10.1186/ s40478-017-0411-2

29. Langston JW, Forno LS, Tetrud J, Reeves AG, Kaplan JA, Karluk D (1999) Evidence of active nerve cell degeneration in the substantia nigra of humans years after 1-methyl-4-phenyl-1,2,3,6-tetrahydropyridine exposure. Ann Neurol 46:598-605. https://doi.org/10.1002/1531-8249(199910)46:4< 598::AID-ANA7>3.0.CO;2-F

30. Lansdall CJ, Coyle-Gilchrist ITS, Jones PS, Rodríguez PV, Wilcox A, Wehmann E, Dick KM, Robbins TW, Rowe JB (2017) Apathy and impulsivity in frontotemporal lobar degeneration syndromes. Brain 140:1792-1807. https://doi.org/10.1093/brain/awx101

31. Liang CL, Nelson O, Yazdani U, Pasbakhsh P, German DC (2004) Inverse relationship between the contents of Neuromelanin pigment and the vesicular monoamine Transporter-2: human midbrain dopamine neurons. J Comp Neurol 473:97-106. https://doi.org/10.1002/cne.20098

32. Malpetti M, Passamonti L, Rittman T, Jones PS, Vasquez-Rodriguez P, BevanJones WR, Hong YT, Fryer TD, Aigbirhio Fl, O'Brien JT, Rowe J (2019) PET markers of tau and neuroinflammation are co-localized in progressive supranuclear palsy. medRxiv:19010702. https://doi.org/10.1101/19010702

33. Mann DMA, Yates PO, Hawkes J (1983) The pathology of the human locus ceruleus. Clin Neuropathol 2:1-7

34. Marner L, Søborg C, Pakkenberg B (2005) Increased volume of the pigmented neurons in the locus coeruleus of schizophrenic subjects. J Psychiatr Res 39:337-345. https://doi.org/10.1016/j.jpsychires.2004.10.008

35. McGeer PL, Itagaki S, Boyes BE, McGeer EG (1988) Reactive microglia are positive for HLA-DR in the: Substantia nigra of Parkinson's and Alzheimer's disease brains. Neurology. https://doi.org/10.1212/wnl.38.8.1285

36. Mioshi E, Dawson K, Mitchell J, Arnold R, Hodges JR (2006) The Addenbrooke's cognitive examination revised (ACE-R): a brief cognitive test battery for dementia screening. Int J Geriatr Psychiatry 21:1078-1085. https://doi.org/10.1002/gps.1610

37. Mouton PR, Pakkenberg B, Gundersen HJG, Price DL (1994) Absolute number and size of pigmented locus coeruleus neurons in young and aged individuals. J Chem Neuroanat 7:185-190. https://doi.org/10.1016/08910618(94)90028-0

38. Murley AG, Rowe JB (2018) Neurotransmitter deficits from fronto temporal lobar degeneration. Brain 141:1263-1285. https://doi.org/10.1093/brain/aw×327

39. Oh J, Eser RA, Ehrenberg AJ, Morales D, Petersen C, Kudlacek J, Dunlop SR, Theofilas P, Resende EDPF, Cosme C, Alho EJL, Spina S, Walsh CM, Miller BL, Seeley WW, Bittencourt JC, Neylan TC, Heinsen H, Grinberg LT (2019) Profound degeneration of wake-promoting neurons in Alzheimer's disease. Alzheimers Dement 15:1253-1263. https://doi.org/10.1016/j.jalz.2019.06.3916

40. Ohm TG, Busch C, Bohl J (1997) Unbiased estimation of neuronal numbers in the human nucleus coeruleus during aging. Neurobiol Aging 18:393-399. https://doi.org/10.1016/50197-4580(97)00034-1

41. Passamonti L, Lansdall C, Rowe JB (2018) The neuroanatomical and neurochemical basis of apathy and impulsivity in frontotemporal lobar degeneration. Curr Opin Behav Sci 22:14-20. https://doi.org/10.1016/..cobeha.2017.12.015

42. Passamonti L, Rodríguez PV, Hong YT, Allinson KSJ, Bevan-Jones WR, Williamson D, Jones PS, Arnold R, Borchert RJ, Surendranathan A, Mak E, Su L, Fryer TD, Aigbirhio Fl, O'Brien JT, Rowe JB (2018) [11C]PK11195 binding in Alzheimer disease and progressive supranuclear palsy. Neurology 90:e1989e1996. https://doi.org/10.1212/WNL.0000000000005610

43. Rascol O, Sieradzan K, Peyro-Saint-Paul H, Thalamas C, Brefel-Courbon C, Senard JM, Ladure P, Montastruc JL, Lees A (1998) Efaroxan, an alpha-2 antagonist, in the treatment of progressive supranuclear palsy. Mov Disord 13:673-676. https://doi.org/10.1002/mds.870130411

44. Sasaki M, Shibata E, Tohyama K, Takahashi J, Otsuka K, Tsuchiya K, Takahashi S, Ehara S, Terayama Y, Sakai A (2006) Neuromelanin magnetic resonance imaging of locus ceruleus and substantia nigra in Parkinson's disease. Neuroreport 17: 1215-1218. https://doi.org/10.1097/01.wnr.0000227984.84927.a7

45. Steele JC, Richardson JC, Olszewski J (1964) Progressive Supranuclear palsy: a heterogeneous degeneration involving the brain stem, basal ganglia and cerebellum with vertical gaze and Pseudobulbar palsy, nuchal dystonia and dementia. Arch Neurol 10:333-359. https://doi.org/10.1001/archneur.1964. 00460160003001

46. Sterio DC (1984) The unbiased estimation of number and sizes of arbitrany particles using the disector. J Microsc 134:127-136. https://doi.org/10.1111/j.1365-2818.1984. tb02501x

47. Sulzer D, Bogulavsky J, Larsen KE, Behr G, Karatekin E, Kleinman MH, Turro N, Krantz D, Edwards RH, Greene LA, Zecca L (2000) Neuromelanin biosynthesis is driven by excess cytosolic catecholamines not accumulated by synaptic vesicles. Proc Natl Acad Sci U S A 97:11869-11874. https:/doi.org/10.1073/pnas.97.22.11869

48. Theofilas P, Ehrenberg AJ, Dunlop S, Di Lorenzo Alho AT, Nguy A, Leite REP, Rodriguez RD, Mejia MB, Suemoto CK, Ferretti-Rebustini REDL, Polichiso L, Nascimento CF, Seeley WW, Nitrini R, Pasqualucci CA, Jacob Filho W, Rueb U, Neuhaus J, Heinsen H, Grinberg LT (2017) Locus coeruleus volume and cell population changes during Alzheimer's disease progression: a stereological study in human postmortem brains with potential implication for early-stage biomarker discovery. Alzheimers Dement 13:236-246. https:// doi.org/10.1016/J.JALZ.2016.06.2362

49. Tsai RM, Lobach I, Bang J, Whitwell JL, Senjem ML, Jack CR, Rosen H, Miller B, Boxer AL (2016) Clinical correlates of longitudinal brain atrophy in progressive supranuclear palsy. Parkinsonism Relat Disord 28:29-35. https:// doi.org/10.1016/j.parkreldis.2016.04.006

50. Vazey EM, Aston-Jones $G$ (2012) The emerging role of norepinephrine in cognitive dysfunctions of Parkinson's disease. Front Behav Neurosci 6:48. https://doi.org/10.3389/fnbeh.2012.00048 
51. Wakamatsu K, Tabuchi K, Ojika M, Zucca FA, Zecca L, Ito S (2015) Norepinephrine and its metabolites are involved in the synthesis of neuromelanin derived from the locus coeruleus. J Neurochem 135:768-776. https://doi.org/10.1111/jnc.13237

52. Wear HJ, Wedderburn CJ, Mioshi E, Williams-Gray CH, Mason SL, Barker RA, Hodges JR (2008) The Cambridge Behavioural inventory revised. Dement Neuropsychol 2:102-107. https://doi.org/10.1590/s198057642009dn20200005

53. Weinshenker D (2018) Long road to ruin: noradrenergic dysfunction in neurodegenerative disease. Trends Neurosci 41:211-223. https://doi.org/10. 1016/j.tins.2018.01.010

54. Whitwell JL, Tosakulwong N, Schwarz CG, Botha H, Senjem ML, Spychalla AJ, Ahlskog JE, Knopman DS, Petersen RC, Jack CR, Lowe VJ, Josephs KA (2019) MRI outperforms [18F]AV-1451 PET as a longitudinal biomarker in progressive Supranuclear palsy. Mov Disord 34:105-113. https://doi.org/10. 1002/mds. 27546

55. Williams DR, Lees AJ (2009) Progressive supranuclear palsy: clinicopathological concepts and diagnostic challenges. Lancet Neurol 8 : 270-279. https://doi.org/10.1016/S1474-4422(09)70042-0

56. Wilson RS, Nag S, Boyle PA, Hizel LP, Yu L, Buchman AS, Schneider JA, Bennett DA (2013) Neural reserve, neuronal density in the locus ceruleus, and cognitive decline. Neurology 80:1202-1208. https://doi.org/10.1212/ WNL.0b013e3182897103

57. Ye Z, Altena E, Nombela C, Housden CR, Maxwell H, Rittman T, Huddleston C, Rae CL, Regenthal R, Sahakian BJ, Barker RA, Robbins TW, Rowe JB (2015) Improving response inhibition in Parkinson's disease with Atomoxetine. Biol Psychiatry 77:740. https://doi.org/10.1016/J.BIOPSYCH.2014.01.024

58. Zecca L, Stroppolo A, Gatti A, Tampellini D, Toscani M, Gallorini M, Giaveri G, Arosio P, Santambrogio P, Fariello RG, Karatekin E, Kleinman MH, Turro N, Hornykiewicz O, Zucca FA (2004) The role of iron and molecules in the neuronal vulnerability of locus coeruleus and substantia nigra during aging. Proc Natl Acad Sci U S A 101:9843-9848. https://doi.org/10.1073/pnas. 0403495101

59. Zhang W, Phillips K, Wielgus AR, Liu J, Albertini A, Zucca FA, Faust R, Qian SY, Miller DS, Chignell CF, Wilson B, Jackson-Lewis V, Przedborski S, Joset D, Loike J, Hong JS, Sulzer D, Zecca L (2011) Neuromelanin activates microglia and induces degeneration of dopaminergic neurons: implications for progression of parkinson's disease. Neurotox Res 19:63-72. https://doi.org/ 10.1007/s12640-009-9140-z

60. Zucca FA, Vanna R, Cupaioli FA, Bellei C, De Palma A, Di Silvestre D, Mauri P, Grassi S, Prinetti A, Casella L, Sulzer D, Zecca L (2018) Neuromelanin organelles are specialized autolysosomes that accumulate undegraded proteins and lipids in aging human brain and are likely involved in Parkinson's disease. npj Park Dis:4. https://doi.org/10.1038/s41531-018-0050-8

\section{Publisher's Note}

Springer Nature remains neutral with regard to jurisdictional claims in published maps and institutional affiliations.

Ready to submit your research? Choose BMC and benefit from:

- fast, convenient online submission

- thorough peer review by experienced researchers in your field

- rapid publication on acceptance

- support for research data, including large and complex data types

- gold Open Access which fosters wider collaboration and increased citations

- maximum visibility for your research: over $100 \mathrm{M}$ website views per year

At $\mathrm{BMC}$, research is always in progress.

Learn more biomedcentral.com/submissions 